\title{
Influencia de la metodología docente en la adquisición rápida de conocimientos
}

\author{
J osep Nebot-Cegarraa, Mercedes Campillo y y orge Pérez \\ a Unitat d'Anatomia i d'Embriologia, Departament de Ciències Morfològiques, Facultat de Medicina, Universitat \\ Autónoma de Barcelona (UAB). ${ }^{\circ}$ Laboratori de Medicina Computacional, U nitat de Bioestadística, Facultat de \\ Medicina, UAB. ${ }^{c}$ Facultat de Ciències de la Salut i de la Vida. Universitat Pompeu Fabra.
}

Introducción. Las evaluaciones de la actividad docente universitaria, por lo general se efectúan con demora respecto al momento en que se han enseñado unos determinados contenidos. Nuestro estudio pretende determinar cómo influye en la adquisición rápida de conocimientos la forma como se transmite la información, así como algunas situaciones ambientales (número de alumnos y horario de la sesión docente)

Material y Métodos. Inmediatamente después de cada sesión de una actividad teoricopráctica se efectuó una prueba de comprensión a 66 alumnos (asignatura Biología del Desarrollo y Teratogenia) Se valoró la existencia de diferencias estadísticamente significativas entre los distintos tipos de cuestiones planteadas. Se analizaron como variables: las características de la metodología docente y del tipo de pregunta; el número de asist entes a cada sesión y el horario.

Resultados: Se obtuvieron mejores resultados: en las cuestiones enseñadas sólo oralmente; en las que requerían del alumno sólo observación y en las que requerían definir conceptos y no identificar y nombrar. Para responder estas últimas la dificultad fue directament e proporcional a la complejidad y la dificultad fonética de los términos correctos. Los asist entes a la primera sesión de la tarde obtuvieron mejores calificaciones que los de la segunda.

Conclusiones: La metodología que permita al alumno percibir la comunicación no verbal del profesor favorece la adquisición rápida del contenido enseñado (transmisión oral a alumnos despreocupados de otras actividades). A corto plazo se obtiene mejor

\footnotetext{
Correspondencia:

Dr. J osep Nebot-Cegarra.

Unitat d'Anatomia i d'E mbriologia,

Departament de Ciències Morfològiques,

Facultat de Medicina, Universitat Autónoma de Barcelona. 08193 Bellaterra (Barcelona)

Correo electrónico: J osep.Nebot@uab.es
}

la capacidad de definir conceptos que la de discriminar casos diferentes, y se recuerdan con mayor precisión los términos menos complejos

Palabras clave:

Educación médica. Evaluación. Aprendizaje.

Comunicación no verbal. Embriología. Docencia universitaria.

The influence of the teaching methodology on the immediate learning.

Introduction: In general, the evaluation of the university students is carried out some days after the lessons were taught. The aim of our study was to establish how much influence the way of transmitting information and other environmental factors (the number of students and the time) have on the immediate learning.

Material and Methods: 66 students on the "Developmental Biology and Teratogeny" course took part in the study. At the end of a theory-practice activity an exam was given based on the matter taught during the session. We were looking for differences in performance based on differences in the teaching method, the type of questions asked, the number of students per session, and the time at which the session was held.

Results: Bett er results were obtained for the content that had been taught without audiovisual support; for the questions for which the students had to define concepts and for the questions that they had to answer with words less complex and with less difficult phonetically. Better results were also obtained by the students who did the session in the first afternoon time slot, in comparison with those who did the session later in the afternoon.

Conclusions: The methodology that enabled the students to perceive the non-verbal communication 
of the teacher (where audiovisual aids were not used) gave better results for immediate learning. The definition of concepts on the part of the students was better that the capacity to discern between them. The less complex terms were retained better than complex ones.

Key words:

Medical education. Evaluation. Learning. Nonverbal

Communication. Embryology. Higher education.

\section{INTRODUCCIÓN}

La actividad docente universitaria, que pretende formar a futuros profesionales, objetiva sus resultados a través de las evaluaciones de las diferentes asignaturas, que por lo general se efectúan con demora respecto al momento en que se han enseñado unos determinados contenidos. Aún desconociendo estadísticas sobre la intención de la mayoría de los docentes, quizás no erraríamos al pensar que una mayoría pretende que su clase o su práctica constituya un reaurso efectivo desde el primer momento, es decir "hay que salir con el tema entendido y casi sabido".

EI procesamiento de la información se efectúa en tres etapas: primero la información entra por los sentidos, sobre todo vista y oído, procesándose como memoria sensorial; en segundo lugar la entrada sensorial que el individuo selecciona como intere sante es transferida a la memoria a corto plazo, donde se retiene brevemente mientras, si procede, se procesa más y se al macena como memoria a largo plazo ${ }^{1}$. De este modo la memoria sensorial y la percepción de que se está ante una cuestión de interés condicionan en principio que los alumnos puedan o no adquirir rápida y suficiente comprensión y retención de un determinado tema. Se apuntan como factores determinantes las características del tema, la capacidad y actitud del alumno y del profesor, así como la metodología utilizada. Asimismo la forma de evaluar, aspecto metodológico, puede ser un factor que matice la valoración del aprendizaje.

Nuestro estudio pretende determinar la influencia que puede tener en la adquisición rápida de conocimientos tanto la forma como se transmite la información, como algunas situaciones ambientales (número de alumnos y horario de la sesión docente) Asimismo se ha valorado la influencia del tipo de preguntas en el resultado.

\section{MATERIAL Y MÉTODOS}

\section{Alumnos: Número y características.}

Participaron en el estudio 66 alumnos de segundo curso de la Licendiatura en Medicina de la Universitat Autònoma de Barcelona, matriculados en la asignatura optativa Biología del Desarrollo y Teratogenia, duranteel curso 1999-00.

\section{Prueba de comprensión.}

Se realizó una pr ueba decomprensión de una actividad teoricopráctica de la mencionada asignatura, en donde se enseñó a los alumnos de forma íntegra l os temas "Gestaciones múl tiples" y "Anomalías placentarias". La prueba se efectuó inmediatamente después definalizar la sesión y se informó de que era voluntaria y sin contingencia en la nota de la asignatura, ya que su valoración con influencia académica se hizo otro día que estaba previamente anunciado.

Los alumnos debían contestar tres preguntas (Fig. 1). En la primera se pedía a qué tipo deter atópagos correspondían cada una de las seis imágenes

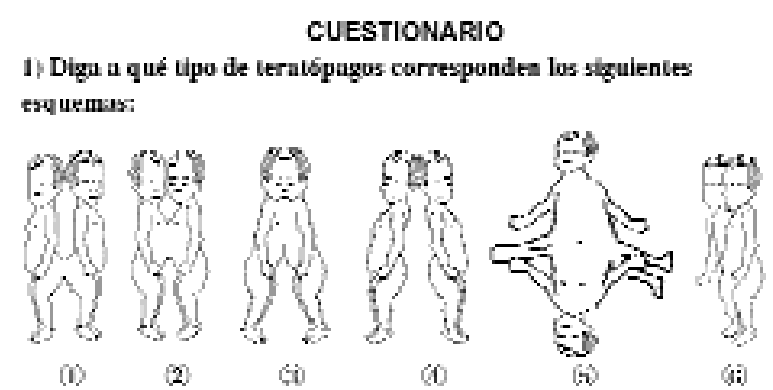

2) Indique bes diferencias entre las placenlas accrela (7), increla (ㄱ) y purrola (9).

3) Indique la secuencia correcta, anotando los nímeros de los pasos adecwados del desarrollo (1- 9) en las tres cuadros en blanca. kisquema de la situación final: 1 placenta; 1 saco corliónleo; 2

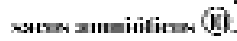
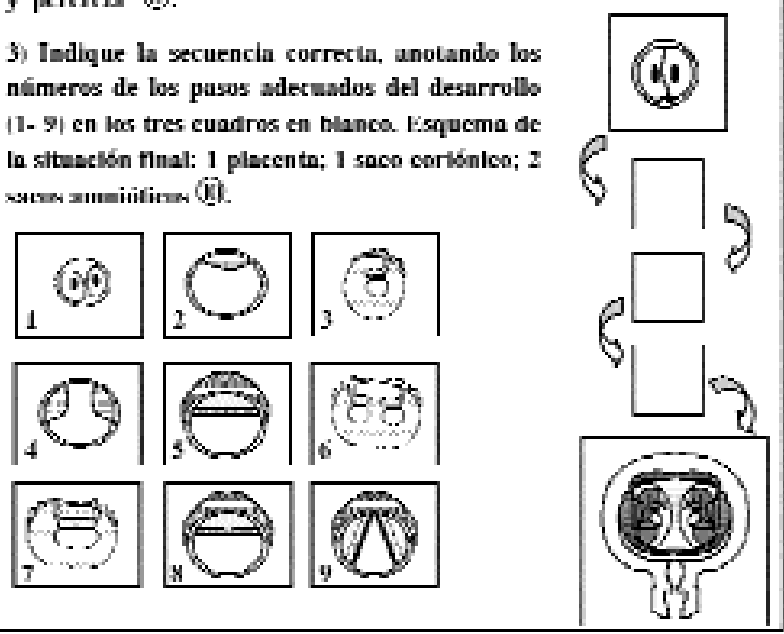

Fig.1 Cuestionario de la prueba de comprensión. Para identificar las cuestiones planteadas en las preguntas, se han añadido números rodeados por círculos. 
representadas (cuestiones 1-6). E n la segunda pregunta debieron indicar las diferencias entre tres tipos de anomalías placentarias cuyos nombres se incluían (cuestiones 7-9). En la tercera pregunta (cuestión 10) debieron indicar sobre una plantilla con tres casillas en blanco, la secuencia cor recta de fases del desarrollo para qué a partir de dos blastómeros sell egara a una gestadi ón gemelar con sendas cavidades amnióticas, una placenta y un saco coriónico. Para resolver esta última cuestión debi eron escoger entre nueve imágenes esquemáticas las tres que eran cor rectas y ordenarlas adecuadamente.

Cada cuestión fue valorada con 1 punto si la respuesta era correcta y 0 puntos si era incorrecta, no contemplándose notas intermedias en las respuestas aproximadas. La nota de la prueba se calculó en una escala de 0 a 10. La puntuación por tipo de cuestión consistió en la suma de los valores obtenidos en las cuestiones correspondientes a las diferentes tipologías y su posterior transformación a una escala de 0 a 10 puntos.

\section{PROCEDIMIENTOS}

1. Organización. Los alumnos estaban agrupados en grupos reducidos entre 4 y 10 personas, siendo convocados según el grupo en primera (de 15 a 17 horas) o en segunda sesión (de 17 a 19 horas).

2. Metodología docente. La actividad teoricopráctica fue impartida siempre por el mismo profesor (J NC) Sus objetivos fueron: 1) que los alumnos conocieran el origen de las gestaciones múltiples, sus anomalías y fueran capaces de deducir sus distintas vías de desarrollo, y 2) que conocieran y supi eran distinguir las diferentes anomalías de la placenta.

Seempl eó una metodol ogía mixta, habiendo aspectos que fueron informados y otros en que se plantearon problemas que el alumno debía resolver aplicando criterios de reflexión que fueron fomentados.

La actividad docente se dividió en varias partes:

a) Gestaciones múlti ples. Se infor mó del concepto, causas conocidas y tipos de gestaciones múltiples, y se plantear on a los alumnos dos tipos de problemas: i) Averiguar cómo durante la gestación de gemel os se llegaba a formar o no la duplicación de la placenta, de la cavidad amniótica y del saco coriónico, debiendo relacionar estos resultados con el momento en que se generaban los dos embriones; ii) Deducir el origen y consecuencias del síndrome de transfusión fetal entregemelos. Este segundo problema no generó ninguna pregunta del cuestionario, mientras que el primero fue objeto de la pregunta 3. Para resolver el primer problema se diseñó una plantilla consistente en una serie de recuadros, unidos mediante flechas indicativas de cada vía de desarrollo. Los recuadros situados en los extremos de las diferentes vías estaban completados con las imágenes oportunas, mientras que el resto estaban en blanco. La resolución del ejercicio consistió en distribuir sobre dichas casillas trece imágen es recortadas que debían corresponder a las secuencias correctas. Un extracto puede verse en la Figura 1.

b) Teratópagos. A los alumnos se les entregó un texto escrito sobre el concepto y dasificación de los teratópagos, incluyéndose imágenes de al gunos tipos. A continuación seprocedióa la explicadión desu contenido siguiendo el citado texto. Asimi smo se proyectaron las imágenes de tres de los ocho tipos explicados y se mostró en la Sala de disección un ejemplar deun cuarto tipo. En consecuencia hubo cuatro tipos del os que no se mostró ninguna imagen. La pregunta 1 estaba basada en este apartado (Fig.1).

c) Anomalías placentarias. Se informó oral e iconográficamente de tres tipos de anomalías placentarias, y solamente de forma oral de otros tres tipos que fueron objeto de la pregunta 2 (Fig. 1). En la Sala de disección los alumnos observaron y palparon una placenta normal y vieron su situación intrauterina.

3. Tipología de las cuestiones (Tabla 1) Para realizar su valoración las cuestiones se agruparon según la forma cómo el alumno recibió la información en la sesión docente y según el tipo de respuesta solicitada en la pregunta que estaba incluida la cuestión: a) según la vía de percepción las cuestiones se agruparon en cuestiones de percepción auditiva (cuestiones 1, 4 y 7-9) en las que el profesor hizo la explicación sin ayuda de iconografía, y de percepción mixta (cuestiones 2,3 y 5-6) en que sí hubo soporte gráfico; b) según la actividad mínima requerida de forma implícita al alumno, se agruparon en cuestiones de observación (cuestiones 1-6) (solamente mirar y/o oír) y cuestiones de acción personal (cuestiones 7-10) (habían de deducir secuencias del desarrollo o debían anotar datos, además de observar); c) según el tipo de acción solicitada en el enunciado de la pregunta se consideraron cuestiones con respuestas de nombrar (cuestiones 1-6), de definir (cuestiones 7-9) y de secuenciar (cuestión10); d) según que el término a nombrar fuese una palabra con uno o dos prefijos se denominaron cuestiones con respuesta de nombrar simples (cuestiones 1 y 46) o complejas (cuestiones 2 y 3); e) Puesto que el sufijo de estos términos fue siempre el mismo, se 
Tabla 1. Tipología de las cuestiones

\begin{tabular}{|c|c|c|c|c|c|c|c|}
\hline \multirow[t]{3}{*}{ Cuestióna } & \multicolumn{4}{|c|}{ Según la recepción de la información } & \multicolumn{3}{|c|}{ Según la acción solicitada en la respuesta } \\
\hline & \multicolumn{2}{|c|}{ Tipo de percepción } & \multicolumn{2}{|c|}{ Actitud mínima requerida } & \multirow[b]{2}{*}{ Nombrarb } & \multirow[b]{2}{*}{ Definir } & \multirow[b]{2}{*}{ Secuenciar } \\
\hline & Auditiva & Visual & Observación & $\begin{array}{c}\text { Acción } \\
\text { personal }\end{array}$ & & & \\
\hline 1 & $x$ & & $x$ & & Pigópago (TS, DFA) & & \\
\hline 2 & $x$ & $\mathrm{X}$ & $\mathrm{x}$ & & Toracoonfalópago (TC, DFM) & & \\
\hline 3 & $\mathrm{X}$ & $\mathrm{X}$ & $\mathrm{X}$ & & Cefalotoracópago (TC, DFB) & & \\
\hline 4 & $x$ & & $x$ & & Craniópago (TS, DFB) & & \\
\hline 5 & $\mathrm{x}$ & $x$ & $x$ & & Isquiópago (TS, DFM) & & \\
\hline 6 & $x$ & $x$ & $x$ & & & & \\
\hline 7 & $x$ & & & $x$ & & $x$ & \\
\hline 8 & $x$ & & & $x$ & & $x$ & \\
\hline 9 & $x$ & & & $x$ & & $x$ & \\
\hline 10 & & $x$ & & $x$ & & & $x$ \\
\hline
\end{tabular}

a Ver figura 1; ${ }^{\mathrm{b}}$ respuesta correcta (complejidad del término, dificultad fonética) DFA, dificultad fonética alta; DFB, dificultad fonética baja; DFM, dificultad fonética media; TC, término complejo; TS, término simple.

\begin{tabular}{|c|c|c|c|c|c|}
\hline & Media $^{b}$ & Desviación Típica & \multicolumn{3}{|c|}{ Percentiles } \\
\hline $\begin{array}{l}\text { Tipo de percepción } \\
\text { Mixta } \\
\text { Auditiva } \\
\text { Actitud mínima requerida } \\
\text { Observación } \\
\text { Acción personal }\end{array}$ & $\begin{array}{l}3.5 \\
5.5 \\
5.2 \\
5.7\end{array}$ & $\begin{array}{l}2.5 \\
2.9 \\
\\
3.6 \\
3.9\end{array}$ & $\begin{array}{l}2.5 \\
4.0 \\
\\
3.8 \\
3.3\end{array}$ & $\begin{array}{l}2.5 \\
6.0 \\
5.0 \\
6.7\end{array}$ & $\begin{array}{l}5.0 \\
8.0 \text { ** } \\
10.0 \\
10.0 \text { n.s. }\end{array}$ \\
\hline $\begin{array}{l}\text { Actitud mínima requerida } \\
\text { Acción personal } \\
\text { Observación } \\
\text { Percepción mixta } \\
\text { Percepción auditiva }\end{array}$ & $\begin{array}{l}4.0 \\
6.3 \\
3.5 \\
5.2\end{array}$ & $\begin{array}{l}2.4 \\
3.3 \\
2.5 \\
3.6\end{array}$ & $\begin{array}{l}1.7 \\
2.5 \\
2.5 \\
3.8\end{array}$ & $\begin{array}{l}3.3 \\
7.5 \\
2.5 \\
5.0\end{array}$ & $\begin{array}{c}5.0 \\
10.0^{* *} \\
5.0 \\
10.0^{* *}\end{array}$ \\
\hline $\begin{array}{l}\text { Acción solicitada en la respuesta } \\
\text { Definir } \\
\text { Nombrar } \\
\text { Complejidad del término } \\
\text { Simple } \\
\text { Complejo } \\
\text { Dificultad fonética } \\
\text { Alta } \\
\text { Media } \\
\text { Baja }\end{array}$ & $\begin{array}{l}5.7 \\
4.0 \\
\\
4.4 \\
3.3 \\
\\
2.7 \\
3.4 \\
6.1\end{array}$ & $\begin{array}{l}3.9 \\
2.4 \\
\\
2.8 \\
3.2 \\
\\
3.3 \\
3.4 \\
3.3\end{array}$ & $\begin{array}{l}3.3 \\
1.7 \\
\\
2.5 \\
0.0 \\
\\
0.0 \\
0.0 \\
5.0\end{array}$ & $\begin{array}{l}6.7 \\
3.3 \\
\\
5.0 \\
5.0 \\
\\
0.0 \\
5.0 \\
5.0\end{array}$ & $\begin{array}{c}10.0 \\
5.0^{*} \\
5.6 \\
5.0^{*} \\
5.0 \\
5.0 \\
10.0^{* *}\end{array}$ \\
\hline
\end{tabular}

${ }^{a}$ No se considera la cuestión $10 ;{ }^{b}$ Puntuación obtenida en escala de $0-10 .{ }^{*} p<0,05 ;{ }^{* *} p<0.001$. ns, no diferencias significativas

consideraron cuestiones de dificultad fonética baja (cuestiones 3 y 4) si la respuesta correcta correspondía a una palabra con prefijo/s de raíz/ces que el alumno conocía previamente y era de fácil relación con el concepto; de dificultad media (cuestiones 2 y 5) si aún conociendo la/s raíz/ces era más difícil su relación conceptual, y de dificultad alta (cuestiones 1 y 6) si la raíz era desconocida por el alumno. Se consideró que los alumnos conocían previamente la raíz de un término si habían tenido ocasión de utilizarla repetidamente con anterioridad en alguna asignatura de la licenciatura. 
4. Valoración de los resultados. Se basó en la determinación de diferencias significativas entre las puntuaciones obtenidas en los diferentes tipos de cuestiones y en los diferentes grupos de alumnos. La cuestión número 10 sól o se tuvo en cuenta en el análisis sobre la actitud mínima requerida, ya que en el análisis según el tipo de percepción y según la acción solicitada en la respuesta, por corresponder a una categoría con una única cuestión, generaba resultados bimodales con únicamente 0 ó 10 puntos como resultados posibles (ver Tabla 1).

Se analizó si había diferencias estadísticamente significativas entre las puntuaciones obtenidas al comparar las cuestiones según el tipo de percepción, la actitud mínima requerida y el tipo de acción solicitada en la respuesta (Tabla 2). Además se analizó si existían diferencias significativas entre las notas de la prueba obtenida por los diferentes grupos de alumnos (Tabla 3). Para este fin se agruparon a los alumnos atendiendo al número de asistentes a cada sesión y al horario de la misma.

5. Análisis estadístico. Las hipótesis de normalidad se contrastaron con el test de KolmogorovSmirnov con la aproximación de Lilliefors. Cuando la comparación de las puntuaciones obtenidas según el tipo de cuestión se refería a dos grupos, se utilizó el test de la t de Student de datos apareados bajo el supuesto de normalidad o la prueba de los rangos con signo de Willcoxon en caso contrario; la comparación de más de dos grupos se realizó con un análisis de la varianza de medidas repetidas o la prueba de Friedman para valores normales o no normales, respectivamente. Los contrastes por grupo de alumnos se efectuó con una t de Student de datos independientes (análisis según la sesión) y con un análisis de la varianza de un factor (análisis según el número de asistentes a la sesión docente) Todos los análisis fueron realizados con el programa estadístico SPSS 10.0 SPSS 10.0. SPSS I nc, Chicago, Illinois 60606\}. El nivel de significación del estudio fue del $5 \%$.

\section{RESULTADOS}

\section{Según la recepción de la información}

Los datos estadísticos se muestran en la Tabla 2.

1. Según la percepción. Se obtuvieron mejores resultados en las cuestiones de percepción auditiva que en las de percepción mixta, siendo las diferencias estadísticamente significativas.

2. Según la actitud mínima requerida al alumno. Se obtuvieron mejores resultados en las cuestiones de observación que en las de acción personal, siendo las diferencias estadísticamente significativas.

3. Según la percepción y la actitud mínima requerida. Cuando se analizaron sólo las cuestiones que requerían una actitud mínima de observación por parte del alumno, se demostró que se obtuvieron significativamente mejores resultados en aquellas en que la información se percibió sólo auditivamente que en aquellas que también se percibió visualmente (percepción mixta).

\section{Según el tipo de respuesta solicitada}

Los datos estadísticos se muestran en la Tabla 2.

1. Según la acción a realizar. Se obtuvi eron significativamente mejores resultados en las respuestas que requirieron definir conceptual mente anomalías placentarias que aquellas donde se pidió identificar por su nombre unas imágenes deteratópagos.

2. Según el tipo de término a nombrar. En las cuestiones con respuestas que requirieron utilizar términos simples (sólo un prefijo) para identificar los tipos de teratópagos, los resultados fueron significativamente mejores que los obtenidos en las cuestiones que se resolvían con términos complejos (con dos prefijos).

3. Según la dificultad fonética del término a nombrar. En cuestiones con respuestas que requirieron utilizar términos de dificultad fonética baja para identificar teratópagos, los resultados fueron signi ficativamente mejores que los obtenidos en las cues-

Tabla 3. Resumen de los resultados estadísticos según el tipo de grupo

\begin{tabular}{|c|c|c|c|}
\hline & $\mathbf{N}$ & Media $^{a}$ & Desviación típica \\
\hline $\begin{array}{l}\text { Número de alumnos presentes en la sesión } \\
\qquad \begin{array}{l}<=7 \\
>7\end{array} \\
\text { Sesión }\end{array}$ & $\begin{array}{l}30 \\
36 \\
\\
43 \\
23\end{array}$ & $\begin{array}{l}5.3 \\
4.7 \\
\\
\\
5.4 \\
4.2\end{array}$ & $\begin{array}{l}2.3 \\
2.2 \text { n.s. } \\
2.4 \\
1.8 \text { * }\end{array}$ \\
\hline
\end{tabular}

${ }^{a}$ Puntuación global obtenida en escala de $0-10 .{ }^{*} p<0.05$. ns, no diferencias significativas 
tiones que se basaban en términos de dificultad fonética media o alta.

\section{Según el tipo de grupo}

Los datos estadísticos se muestran en la Tabla 3.

No se encontraron diferencias estadísticamente significativas en cuanto a los resultados de la evaluación según el número de alumnos presentes en las distintas sesiones.

Los alumnos que asistieron a la primera sesión de la activi dad teoricopráctica obtuvier on significativamente mejores resultados que los de la segunda.

\section{DISCUSIÓN}

Nuestro estudio ha sido planeado para ver qué influencia tiene la metodología docente sobre la adquisición de conocimientos sin que medie la posibilidad del estudio personal. Por este motivo se efectuó una prueba de comprensión inmediatamente después de que se enseñara a los alumnos los temas objeto de evaluación, utilizando diversos métodos. Para no desvirtuar nuestro objetivo, se eligieron en la prueba temas que eran totalmente nuevos para los alumnos, ya que únicamente se les enseñó en la actividad docente que precedió a la evaluación.

Los resultados obtenidos muestran que en la adquisición rápida de conocimientos ha resultado más efectiva la explicación oral sin iconografía, que si se acompaña de ella (Tabla 2). Estos datos resultan cuanto menos sorprendentes, ya que al tratarse de temas de unas ciencias morfológicas (embriología y teratogenia) se podría presuponer que la imagen tendría más contingencia en el aprendizaje precoz. En las cuestiones que se explican oralmente y con imágenes, y por tanto son percibidas auditiva y visualmente, el alumno puede, además de escuchar, observar al profesor, ver las imágenes proyectadas o, en su caso, inspeccionar la preparación anatómica. Cuando estos complementos visuales no están presentes el alumno puede centrar su atención en el profesor y por tanto apreciar toda la gestualidad que este imprime en su explicación, siendo éste un hecho didáctico diferencial que destacamos como favorable. Quizás para algunos alumnos las imágenes visuales puedan tener un efecto de distracción mayor que de ilustración ${ }^{2}$.

Otros resultados apuntan en el mismo sentido, ya que se ha determinado que mantener una actitud activa, como tomar apuntes o resolver un problema, no favorece tanto el aprendizaje rápido como mantener una actitud de observación pura. La toma de apuntes tiene como principal finalidad poder disponer de una información tratada personalmente ${ }^{3}$, aunque para algunos alumnos la preocupación por la literalidad de lo an otado favorezca más su estudio en otro momento que la comprensión inmediata de la materia explicada ${ }^{4}$ y asimismo impida apreciar los matices expresivos del docente. Incluso cuando al alumno se le había entregado previamente documentación sobre las cuestiones que se iban a explicar y, en consecuencia, no debía hacer otra cosa que escuchar y observar, Ia fijación rápida de conceptos resultó mejor cuando no se utilizó iconografía, es decir cuando pudieron observar todo el tiempo al profesor. Además la expresi ón no verbal, como movimi entos de la cabeza y de las manos, que se efectúan mientras se habla, favorecen la fluidez y la amplitud del léxico, tal como determino experimentalmente en 1931 Dobrogaev (citado por Mc Clave ${ }^{5}$ ).

El enseñante basa su eficacia didáctica en su daridad expositiva, en la habilidad por gestionar la interacción en el aula y en su capacidad por "seducir" a los alumnos ${ }^{6}$. A la vista de nuestros resultados, que la mirada de los estudiantes se fije sobre el profesor y aprecie su lenguaje no verbal tiene también contingencia positiva en la adquisición rápida de conocimientos.

Hemos determinado que el tipo de respuesta requeri da infl uye significativamente en el resul tado de la evaluación del aprendi zaje rápido, ya que se respondieron mejor las cuestiones en que se debía definir conceptos que aquellas en que se debía i dentificar por su nombre diversas mal formaciones representadas en imágenes (Tabla 2). Debido a que en todos los casos el objetivo ha sido enseñar conceptos, los alumnos debían seguir un proceso mental más homogén eo cuando debieron dar una respuesta de defini ción conceptual que en el caso de tener que identificar. A pesar de que ninguno de los conceptos enseñados resul taba abstracto, en las respuestas de identificar los alumnos debían evocar "su concepto" a partir de imágenes concretas y traducirlo en nomenclatura adecuada, mi entras que en el caso de tener que definir conceptos ya se aportaban en el enunciado los nombres que debían provocar su evocación y a continuación debían explicarlos.

Uno de los cambios más importantes que experimenta un estudiante de medicina en su transformación en experto, es la adquisición de terminología profesional ${ }^{7}$. El grado de dificultad en el aprendiza- 
je de los términos es heterogéneo y la velocidad de los procesos mentales implicados en su adquisición depende de las posibilidades de relacionarlos con preconceptos ${ }^{8}$. Nuestros datos están en consonancia con estos principios ya que se ha demostrado que a corto plazo se aprenden mejor las palabras que sean estructuralmente más simples y muy significativas para el alumno.

Hemos valorado también si hay situadiones ambientales que puedan influir en el aprendizaje rápido. Se ha observado que los alumnos que efectuaron la actividad docente entre las 15 y las 17 horas obtuvieron resultados estadísticamente mejores que aquellos que la realizaron entre las 17 y 19 horas (Tabla 3). Este dato apunta a que el factor cansancio pueda ser la explicación, y en este caso sería un factor más fuerte que el factor ligado al ritmo circadiano de la atención, ya que el horario de la primera sesión coincidía con una de las fases diarias de menor activación de la atención. En cambio, el mayor o menor número de alumnos en las sesiones no condicionó diferencias estadísticamente significativas en el grado de aprendizaje (Tabla 3).

En nuestro estudio se han comparado los resultados obtenidos en las evaluaciones de cuestiones distintas enseñadas con métodos diversos. Para precisar mejor la influencia de cada uno de estos métodos en el aprendizaje rápido será necesario en un futuro comparar los resultados obtenidos al explicar un mismo tema mediante métodologías diversas, pero necesariamente a grupos de alumnos diferentes. No obstante, de los datos obtenidos en este trabajo se desprenden varias sugerencias de cómo mejorar la actividad del docente, ya que aunque se ha valorado únicamente su influencia en el aprendizaje a corto plazo, no cabe duda de que ésta representa el punto de partida de la posterior consolidación de los conocimientos.

\section{BIBLIOGRAFIA}

1. Good Th., Brophy P. Psicologia educativa contemporánea. México DF : Mc Graw-Hill Interamericana Editores, 1996.

2. Coniam D. The use of audio or video comprehension as an assessment instrument in the certification of English language teachers: a case study. System 2001; 29:1-14.

3. Hoffbeck G., Walter J. ¿Cómo tomar apuntes?. Bilbao: Deusto DL, 1988.

4. Castelló $M$, Monereo $C$. Com prenen apunts els estudiants?. Docència de Qualitat (Estudis i recerques) UAB 1999; ficha no 4.

5. Mc Clave EZ. Linguistic functions of head movements in the context of speech. J Pragmat 2000; 32:855-878.

6. Cros Alavedra A. La primera classe d'un curs. Docència de Qualitat (Bones practiques) UAB 1999; ficha $\mathrm{n}$ o 6 .

7. Lucas P., Lenstrup M., Prinz J ., Williamson D., Yip H. Y Tipoe G. (1977) Language as a barrier to the acquisition of anatomical knowledge. Med Educ 31:81-86.

8. Meyer DE, Schvaneveldt RW. Meaning, memory structure, and mental processes. En: Cofer ChN, editor. The structure of human memory. San Francisco: Freeman WH \& Co., 1976; 54-89. 